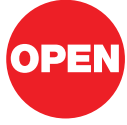

SUBJECT AREAS:

ANIMAL BEHAVIOUR

BEHAVIOUR

ANIMALS

ZOOLOGY

Received

14 July 2011

Accepted

28 September 2011

Published

3 November 2011

Correspondence and requests for materials should be addressed to

T.G. (tg227@st-and. ac.uk)

\section{Community-specific evaluation of tool affordances in wild chimpanzees}

\author{
Thibaud Gruber ${ }^{1,2}$, Martin N. Muller ${ }^{3,6}$, Vernon Reynolds ${ }^{2,4}$, Richard Wrangham ${ }^{5,6}$ \& Klaus Zuberbühler ${ }^{1,2}$
}

\begin{abstract}
${ }^{1}$ School of Psychology, University St Andrews, St Andrews, KY16 9JP, Scotland, United Kingdom, ${ }^{2}$ Budongo Conservation Field Station, Masindi, Uganda, ${ }^{3}$ Department of Anthropology, University of New Mexico, MSC01-1040, Albuquerque, NM 87131 , U.S.A., ${ }^{4}$ Magdalen College, Oxford University, Oxford, OXI 4AU, England, United Kingdom, ${ }^{5}$ Department of Anthropology, Harvard University, 11 Divinity Avenue, Cambridge, MA 02138, U.S.A., ${ }^{6}$ Kibale Chimpanzee Project, c/o MUBFS, PO Box 409, Fort Portal, Uganda.
\end{abstract}

The notion of animal culture, defined as socially transmitted community-specific behaviour patterns, remains controversial, notably because the definition relies on surface behaviours without addressing underlying cognitive processes. In contrast, human cultures are the product of socially acquired ideas that shape how individuals interact with their environment. We conducted field experiments with two culturally distinct chimpanzee communities in Uganda, which revealed significant differences in how individuals considered the affording parts of an experimentally provided tool to extract honey from a standardised cavity. Firstly, individuals of the two communities found different functional parts of the tool salient, suggesting that they experienced a cultural bias in their cognition. Secondly, when the alternative function was made more salient, chimpanzees were unable to learn it, suggesting that prior cultural background can interfere with new learning. Culture appears to shape how chimpanzees see the world, suggesting that a cognitive component underlies the observed behavioural patterns.

eports of large-scale community-specific behavioural differences in wild chimpanzees have led to the provocative hypothesis that there is no fundamental difference in kind between chimpanzee and human cultures $^{1-5}$. This conclusion has remained highly controversial, for both empirical and theoretical reasons. The claim is largely based on a systematic analysis of over 150 years of observational data from seven long-term chimpanzee field sites, but this research has sometimes been dismissed as mere "discussions" "6-7 rather than direct evidence for culture in chimpanzees. Although the community-specific behavioural diversity in chimpanzees is generally accepted, sceptics continue to argue that this diversity could be the product of individual trial-and-error learning in response to the specific ecological conditions present at the different field sites. If this were the case, then this community-specific behavioural diversity would not qualify as 'cultural' in the human sense and the two phenomena would merely be analogous ${ }^{6,8-9}$. For instance, some authors have claimed that an infant chimpanzee observing how wind can shift vegetation and expose ants may learn as much as if it had witnessed its mother doing so and subsequently develop the behaviour on its own through a learning mechanism called 'emulation' ${ }^{10-11}$. If such reasoning was true, it would have to be concluded that social variables are irrelevant. Although the term 'emulation' has been used in other ways since first proposed by Tomasello and colleagues ${ }^{12}$, the key point is that animals may simply learn to reproduce the result of an action rather than the action itself $f^{8,11}$. Additionally, the way this phenomenon may occur appears to grant a key role to the ecological surroundings in generating the learning of novel behaviours. For instance, Tennie al. ${ }^{8}$ state that "nut crackers and termite fishers leave their tools and detritus behind, and in the right place, which makes the learning of their offspring and others much easier" (p. 2406). Emulation has been investigated extensively in captivity, notably through so-called 'ghost' experiments, in which the novel behaviour is demonstrated without any relevant social input, and in some cases this has been sufficient for chimpanzees to learn novel behaviours ${ }^{13-14}$. However, in some other experiments involving complex tool use, learning was not observed, which was attributed to the absence of a social model ${ }^{15}$.

What social learning mechanism chimpanzees rely on in the wild is unknown. In current discussions, the consensus is that chimpanzees have access to a range of learning mechanisms, including emulation and imita$\operatorname{tion}^{16}$. Others have argued that there is no strong dichotomy between individual and social learning but that both mechanisms are necessary in the acquisition of cultural behaviours, as addressed by the master-apprentice ${ }^{17}$ or the hybrid-learning ${ }^{18}$ models. Tennie et al.'s model ${ }^{8}$ suggests that the acquisition of novel behaviours is influenced by what individuals encounter in their environment, notably artefacts left behind by other individuals, but that demonstration is not necessary. According to this view the ecological settings or 'affordances' (defined as 
"physical properties of objects and of relationships among objects"19) may be enough to select for behaviours used by a chimpanzee to deal with a task. In recent experiments, however, we found that different Ugandan chimpanzee communities of the same subspecies, Pan troglodytes schweinfurthii, reacted differently to the same apparatus, a rectangular-shaped cavity drilled into a fallen horizontal log that was filled with liquid honey. Individuals of the Sonso community of Budongo Forest probed the cavity with their fingers or manufactured leaf-sponges to use as probes while individuals of the Kanyawara community of Kibale National Park manufactured sticks to extract the hone ${ }^{20}$. These results thus suggest that chimpanzee behaviour is not just driven by the affordances of a task. Results were more consistent with the hypothesis that community-specific tool choices are the result of pre-existing cultural differences in tool use behaviour ${ }^{21}$. Additionally, we found that chimpanzees in the two communities differed in their propensity to resort to tool use: in Kanyawara, only 3 of 17 individuals tested did not manufacture a stick to access honey, while in Sonso only 4 of 24 manufactured a leaf-sponge. Although manufacturing a tool was not required in the first experiment as most honey was accessible with fingers, it was mandatory in the second experiment (obligatory condition), as the honey was too far to reach with fingers. Nonetheless, only 2 Sonso individuals who participated in this experiment produced a tool (a leaf-sponge); the 9 others remained unsuccessful.

Here, we investigated if chimpanzees confronted with a novel task perceive an artificially provided tool in community-specific ways, i.e. whether individuals evaluate the tool's affordances in relation to the task through a cultural filter. To address this question, we presented members of the Sonso and Kanyawara community with the same task, liquid honey trapped in a rectangular-shaped hole. In the critical test condition, we provided a suitable tool next to the hole, a $40 \mathrm{~cm}$ branch of an Alstonia shrub, with all leaves removed for half of its length (fig. 1). We were interested in which parts of the tool chimpanzees of the two communities would find suitable to solve the problem. According to Tennie et al.'s model, chimpanzees should react in a species-specific way, as the tools provided, and their affordances, were identical in both communities. According to our previous findings ${ }^{20}$, chimpanzees should react in a communityspecific way, as their assessment of the tool would be biased by their community-specific knowledge.

In a second experiment, we exposed members of the Sonso community to the solution preferred by the Kanyawara individuals, i.e. by letting them encounter and retrieve the leafy stick directly from the

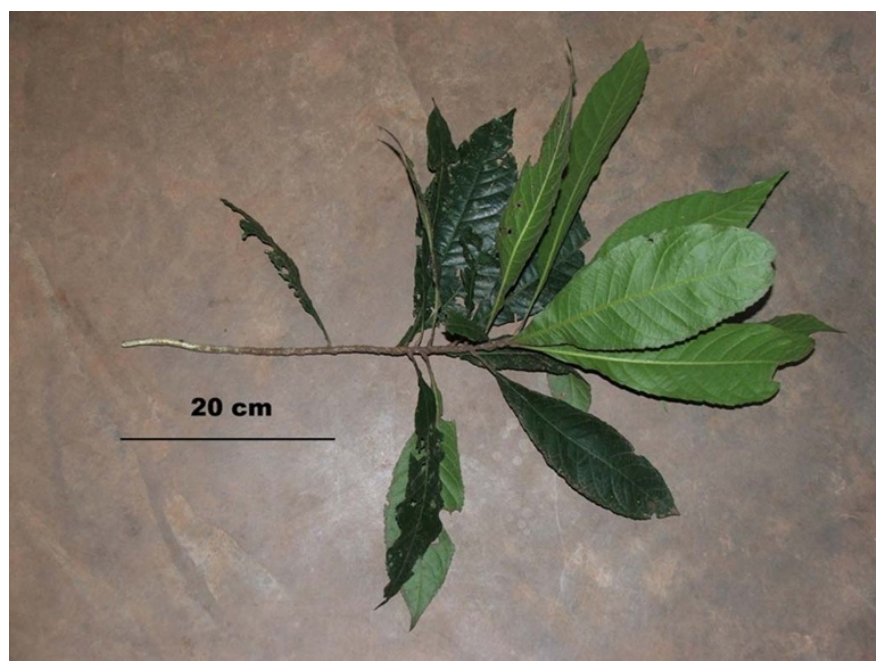

Figure 1 The leafy stick provided during the experiments: a $40 \mathrm{~cm}$ branch of Alstonia ssp, stripped of leaves from the lower $20 \mathrm{~cm}$ of its length. hole, as many of them had still not found a tool-based solution to the honey trap problem. This way, individuals were able to experience directly the physical action of stick use. In 20 years of observations, no Sonso chimpanzee has ever been observed to use a stick in foraging related contexts, although leaf-sponges are fabricated routinely to access water from cavities ${ }^{22}$. This second experiment thus addressed the question of whether chimpanzee will learn a novel behaviour if induced to execute it and, by doing so, to solve the problem on their own.

\section{Results}

Baseline. Although Kanyawara individuals engaged somewhat longer with the apparatus, there was no significant difference in engagement time between Sonso and Kanyawara individuals, suggesting that performance differences could not be ascribed to differences in motivation $\left(\mathrm{N}_{\text {Kanyawara }}=14, \mathrm{~N}_{\text {Sonso }}=29\right.$, Mann-Whitney test, $\mathrm{U}=130 \mathrm{p}=0.058$, excluding QT in Kanyawara as an outlier; table 1 and figure 2 ). $\mathrm{N}=29$ chimpanzees of the Sonso community engaged in one or both experiments, but no one used or produced a stick to access the honey although $\mathrm{N}=7$ manufactured leaf-sponges (2009 study: $\mathrm{N}=4$; this study: $\mathrm{N}=3$ ). The remaining individuals did not produce any tools at all and were thus largely unable to access the honey. The fact that Kanyawara chimpanzees showed a trend towards longer engagement time was probably due to the fact that they were more successful in extracting honey than the Sonso individuals, who abandoned the apparatus earlier (figure 2).

Experiment 1: Provisioning of the leafy stick. In Sonso, 21 individuals participated in the experiment (table 1). Three of $21(14.3 \%)$ seized the leafy stick we had provided. All proceeded to detach the leaves with their lips, discard the stick and roll the leaves in their mouths to produce a sponge (see video 1). The remaining 18 individuals ignored the leafy stick, but two of these (11.1\%) manufactured a leaf-sponge from the surrounding vegetation.

In Kanyawara, 12 individuals participated in the experiment (table 1). 5 of 12 (41.7\%) seized the leafy stick, and all 5 proceeded to insert the bare end of the stick to acquire the honey (see video 2). 3 of 5 removed and discarded the leafy part from the stick before inserting it but none manufactured a leaf sponge. The remaining 7 individuals ignored the tool but 4 of these (57.1\%) manufactured a stick tool from the surrounding vegetation.

Experiment 2: Highlighting the dipping function of the leafy stick (Sonso only). 20 Sonso individuals participated in the experiment in at least one of the conditions. The shared feature of this experiment was that the leafy stick was already inserted into the cavity when individuals arrived at the apparatus, thus making its functional potential as a dipping tool most salient. 15 of 20 individuals (75.0\%) interacted with the leafy stick, but none used the tool to extract honey. Two individuals simply touched the leafy stick but did not retrieve it; three individuals grabbed and retrieved the leafy stick but then discarded it without further signs of interest. 10 removed the leafy stick to engage with the honey-covered part (smell: 6 of 10; consume: 4 of 10; table 2). All individuals subsequently discarded the leafy stick and none used it as a dipping tool. Finally, 5 of the 20 individuals ignored the leafy stick completely, but instead inserted one of their hands into the hole. After these initial interactions, 19 of 20 individuals continued to probe (unsuccessfully) with their hands, or they simply walked away. One individual manufactured a leaf-sponge and succeeded to extract honey.

In sum, although we presented the leafy stick in a way that revealed its functional properties in retrieving honey, either as a brush or as a dipping tool, all the Sonso chimpanzees who engaged in the experiment, including those who (accidentally) obtained honey by removing the leafy stick from the hole, failed to use it in these configurations. None of the non-tool using individuals discovered any of 
Table 1 | Summary of the duration of engagement with the apparatus and use of tools by chimpanzees at Sonso and Kanyawara during baseline and experimental condition 1 .

Baseline $^{a}$

Experiment 1

\begin{tabular}{|c|c|c|c|c|c|c|c|c|c|c|c|c|c|}
\hline Community & ID & Age Class & Gruber etal. 09 (s) & $\#$ & Tool 09 & Baseline 10 (s) & $\#$ & Tool 10 & Total (s) & $\#$ & Exp. Cond. 1 (s) & $\#$ & Tool \\
\hline Kanyawara & $\mathrm{AL}$ & Adult & 10 & 1 & Stick & 128 & 2 & Stick & 138 & 3 & $x$ & $x$ & $x$ \\
\hline Kanyawara & AT & Juvenile & 259 & 2 & Stick & $x$ & $x$ & $X$ & 259 & 2 & $x$ & $\hat{x}$ & $x$ \\
\hline Kanyawara & $A Z$ & Juvenile & $x$ & $x$ & $x$ & 460 & 1 & Stick & 460 & 1 & $x$ & $x$ & $x$ \\
\hline Kanyawara & $\mathrm{BO}$ & Juvenile & 97 & 1 & Stick & $x$ & $x$ & $x$ & 97 & 1 & $x$ & $x$ & $x$ \\
\hline Kanyawara & ES & Sub-Adult & 286 & 1 & Stick & $x$ & $x$ & $x$ & 286 & 1 & $x$ & $x$ & $x$ \\
\hline Kanyawara & EU & Juvenile & 846 & 2 & Stick & $x$ & $x$ & $x$ & 846 & 2 & $x$ & $x$ & $x$ \\
\hline Kanyawara & KK & Adult & $x$ & $x$ & $x$ & 72 & 1 & No & 72 & 1 & $x$ & $x$ & $x$ \\
\hline Kanyawara & LK & Adult & $x$ & $x$ & $x$ & $x$ & $x$ & $x$ & 0 & $x$ & 4 & 1 & No \\
\hline Kanyawara & LR & Adult & 502 & 1 & Stick & $x$ & $x$ & $x$ & 502 & 1 & $x$ & $x$ & $x$ \\
\hline Kanyawara & $N P$ & Sub-Adult & 2899 & 4 & Stick & $x$ & $x$ & $x$ & 2899 & 4 & 347 & 1 & Stick, LS-Stick \\
\hline Kanyawara & $O G$ & Juvenile & 852 & 1 & Stick & 83 & 1 & Stick & 935 & 2 & 95 & 2 & Stick b \\
\hline Kanyawara & От & Sub-Adult & 296 & 3 & Stick & 56 & 1 & Stick & 352 & 4 & 15 & 1 & LS-Stick \\
\hline Kanyawara & OU & Adult & $x$ & $x$ & $x$ & $x$ & $x$ & $x$ & 0 & $x$ & 179 & 1 & Stick \\
\hline Kanyawara & $P G$ & Adult & 6 & 1 & No & 43 & 2 & No & 49 & 3 & 29 & 3 & No \\
\hline Kanyawara & QT & Adult & 7280 & 11 & Stick & $x$ & $x$ & $x$ & 7280 & 11 & 735 & 3 & LS-Stick \\
\hline Kanyawara & TG & Adult & $x$ & $x$ & $x$ & $x$ & $x$ & $x$ & 0 & $x$ & 545 & 4 & Stick \\
\hline Kanyawara & TJ & Adult & 1540 & 1 & Stick & $x$ & $x$ & $x$ & 1540 & 1 & 54 & 1 & Stick \\
\hline Kanyawara & TS & Juvenile & $x$ & $x$ & $x$ & $x$ & $x$ & $x$ & 0 & $x$ & 56 & 1 & No \\
\hline Kanyawara & $\mathrm{TT}$ & Juvenile & $x$ & $x$ & $x$ & 44 & 1 & Stick & 44 & 1 & 3106 & 3 & Stick, LS-Stick \\
\hline Kanyawara & WL & Adult & $x$ & $x$ & $x$ & $x$ & $x$ & $x$ & 0 & $x$ & 649 & 3 & Stick, LS-Stick \\
\hline Sonso & AN & Sub-Adult & 30 & 1 & No & $x$ & $x$ & $x$ & 30 & 1 & $x$ & $x$ & $x$ \\
\hline Sonso & FD & Sub-Adult & $x$ & $x$ & $x$ & 5 & 1 & No & 5 & 1 & $x$ & $x$ & $x$ \\
\hline Sonso & FK & Juvenile & $x$ & $x$ & $x$ & 10 & 1 & No & 10 & 1 & $x$ & $x$ & $x$ \\
\hline Sonso & $\mathrm{HL}$ & Sub-Adult & 98 & $\hat{1}$ & No & $x$ & $x$ & $x$ & 98 & 1 & $\hat{x}$ & $\hat{x}$ & $\hat{x}$ \\
\hline Sonso & $\mathrm{HT}$ & Adult & 102 & 1 & Leaves & $x$ & $x$ & $x$ & 102 & 1 & $x$ & $x$ & $x$ \\
\hline Sonso & $\mathrm{HW}$ & Adult & 100 & 1 & Leaves & 281 & 4 & No & 381 & 5 & 434 & 5 & LS-Sponge \\
\hline Sonso & $\mathrm{JT}$ & Juvenile & $x$ & $x$ & $x$ & 121 & 1 & No & 121 & 1 & 198 & 1 & Leaves \\
\hline Sonso & KA & Juvenile & $x$ & $x$ & $x$ & 20 & 1 & No & 20 & 1 & 274 & 2 & Leaves \\
\hline Sonso & $\mathrm{KE}$ & Sub-Adult & $\hat{x}$ & $\hat{x}$ & $\hat{x}$ & 10 & 1 & No & 10 & 1 & $x$ & $x$ & $x$ \\
\hline Sonso & $\mathrm{KL}$ & Adult & $x$ & $x$ & $x$ & 5 & 1 & No & 5 & 1 & $x$ & $\hat{x}$ & $x$ \\
\hline Sonso & $\mathrm{KM}$ & Juvenile & $x$ & $x$ & $x$ & $x$ & $x$ & $x$ & 0 & $x$ & $x$ & $x$ & $x$ \\
\hline Sonso & $\mathrm{KR}$ & Juvenile & $x$ & $x$ & $x$ & 30 & 1 & No & 30 & 1 & 192 & 3 & No \\
\hline Sonso & KS & Juvenile & $x$ & $x$ & $x$ & 235 & 1 & Leaves & 235 & 1 & $x$ & $x$ & $x$ \\
\hline Sonso & $\mathrm{KT}$ & Adult & $x$ & $x$ & $x$ & $x$ & $x$ & $x$ & 0 & $x$ & 44 & 2 & No \\
\hline Sonso & $\mathrm{KU}$ & Adult & $x$ & $x$ & $x$ & $x$ & $x$ & $x$ & 0 & $\hat{x}$ & $x$ & $x$ & $x$ \\
\hline Sonso & KW & Adult & $x$ & $x$ & $x$ & 78 & 3 & No & 78 & 3 & 83 & 3 & No \\
\hline Sonso & $\mathrm{KY}$ & Adult & $x$ & $x$ & $x$ & 335 & 1 & No & 335 & 1 & 37 & 1 & No \\
\hline Sonso & $\mathrm{KZ}$ & Adult & $x$ & $x$ & $x$ & 225 & 3 & No & 225 & 3 & 40 & 2 & No \\
\hline Sonso & $M L$ & Adult & 45 & 1 & $x$ & $x$ & $x$ & $x$ & 45 & 1 & $x$ & $\bar{x}$ & $x$ \\
\hline Sonso & $M N$ & Juvenile & 45 & 1 & $\hat{x}$ & $x$ & $\hat{x}$ & $\hat{x}$ & 45 & 1 & $\hat{x}$ & $\hat{x}$ & $\hat{x}$ \\
\hline Sonso & MS & Adult & $x$ & $x$ & $x$ & 335 & 1 & No & 335 & 1 & 740 & 4 & No \\
\hline Sonso & NB & Adult & 478 & 1 & No & 1069 & 3 & No & 1547 & 4 & 387 & 5 & No \\
\hline Sonso & NK & Adult & $x$ & $x$ & $x$ & $x$ & $x$ & $x$ & 0 & $x$ & 15 & 1 & No \\
\hline Sonso & $N R$ & Sub-Adult & 62 & 1 & No & 329 & 4 & No & 391 & 5 & 50 & 2 & No \\
\hline Sonso & NT & Juvenile & 493 & 1 & No & 1245 & 4 & Leaves & 1738 & 5 & 685 & 4 & LS-Sponge \\
\hline Sonso & OK & Adult & $x$ & $x$ & $x$ & $x$ & $x$ & $x$ & 0 & $x$ & $X$ & $x$ & $x$ \\
\hline Sonso & PS & Sub-Adult & $x$ & $x$ & Leaves & $x$ & $x$ & $x$ & 0 & $x$ & $x$ & $x$ & $x$ \\
\hline Sonso & $\mathrm{RE}$ & Sub-Adult & $x$ & $x$ & Leaves & $x$ & $x$ & $x$ & 0 & $x$ & 119 & 3 & No \\
\hline Sonso & $\mathrm{RS}$ & Sub-Adult & $x$ & $x$ & $x$ & 1082 & 3 & Leaves & 1082 & 3 & 142 & 1 & LS-Sponge \\
\hline Sonso & $\mathrm{SB}$ & Adult & 75 & 1 & No & 285 & 2 & No & 360 & 3 & $x$ & $x$ & $x$ \\
\hline Sonso & SE & Adult & $x$ & $x$ & $x$ & 45 & 1 & No & 45 & 1 & $x$ & $x$ & $x$ \\
\hline Sonso & $S M$ & Adult & $\hat{x}$ & $\hat{x}$ & $\hat{x}$ & 45 & 1 & No & 45 & 1 & $x$ & $\hat{x}$ & $\hat{x}$ \\
\hline Sonso & $S Q$ & Adult & $x$ & $x$ & $x$ & 34 & 3 & No & 34 & 3 & 74 & 4 & No \\
\hline Sonso & TK & Adult & $x$ & $x$ & $x$ & 267 & 2 & No & 267 & 2 & $x$ & $x$ & $x$ \\
\hline Sonso & VR & Sub-Adult & $x$ & $x$ & $x$ & 115 & 1 & No & 115 & 1 & 233 & 1 & No \\
\hline Sonso & WL & Adult & $x$ & $x$ & $x$ & 102 & 1 & No & 102 & 1 & $x$ & $x$ & $x$ \\
\hline Sonso & ZD & Juvenile & $\hat{x}$ & $x$ & $\hat{x}$ & 33 & 1 & No & 33 & 1 & $\hat{x}$ & $\hat{x}$ & $\hat{x}$ \\
\hline Sonso & $\mathrm{ZF}$ & Adult & $x$ & $x$ & $x$ & 18 & 1 & No & 18 & 1 & 7 & 1 & No \\
\hline Sonso & ZG & Sub-Adult & $x$ & $x$ & $x$ & 119 & 3 & No & 119 & 3 & $x$ & $x$ & $x$ \\
\hline Sonso & ZK & Juvenile & $x$ & $x$ & $x$ & $x$ & $x$ & $x$ & 0 & $x$ & 4 & 1 & No \\
\hline Sonso & $\mathrm{ZL}$ & Adult & $x$ & $x$ & $x$ & 287 & 5 & No & 287 & 5 & 22 & 2 & No \\
\hline Sonso & $Z M$ & Adult & $x$ & $x$ & $x$ & 25 & 2 & No & 25 & 2 & 8 & 2 & No \\
\hline $\begin{array}{l}\text { ''Stick/leaves' } \\
\text { modification; } \\
\text { \#: number of in } \\
\text { X: individual di }\end{array}$ & & & xperiment. & & & . & & & & & and us. & & $\begin{array}{l}\text { r leaves) or no } \\
\text { used without }\end{array}$ \\
\hline
\end{tabular}




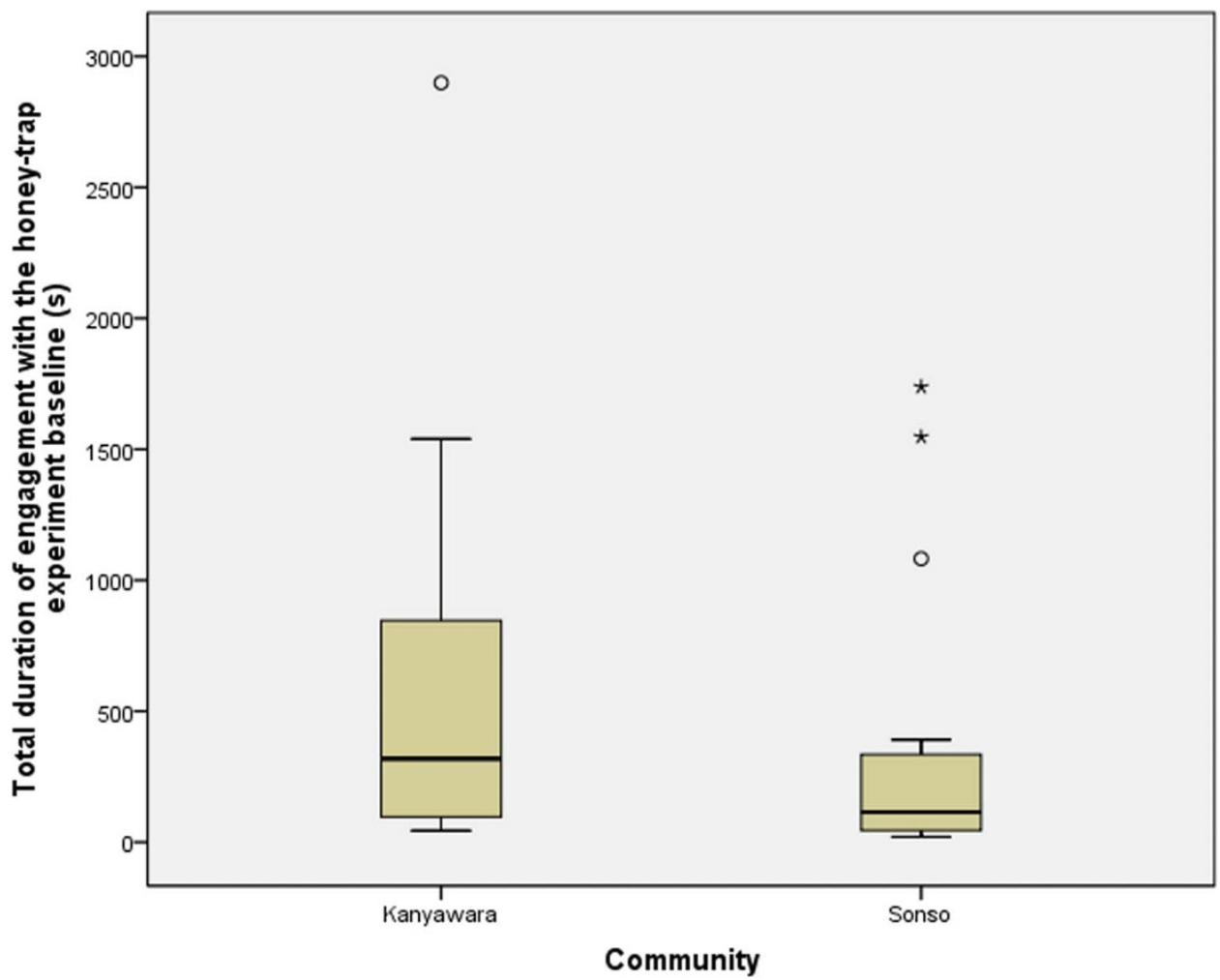

Figure $2 \mid$ Boxplot presenting durations of engagement with the apparatus in Sonso and Kanyawara during the baseline conditions (see text).

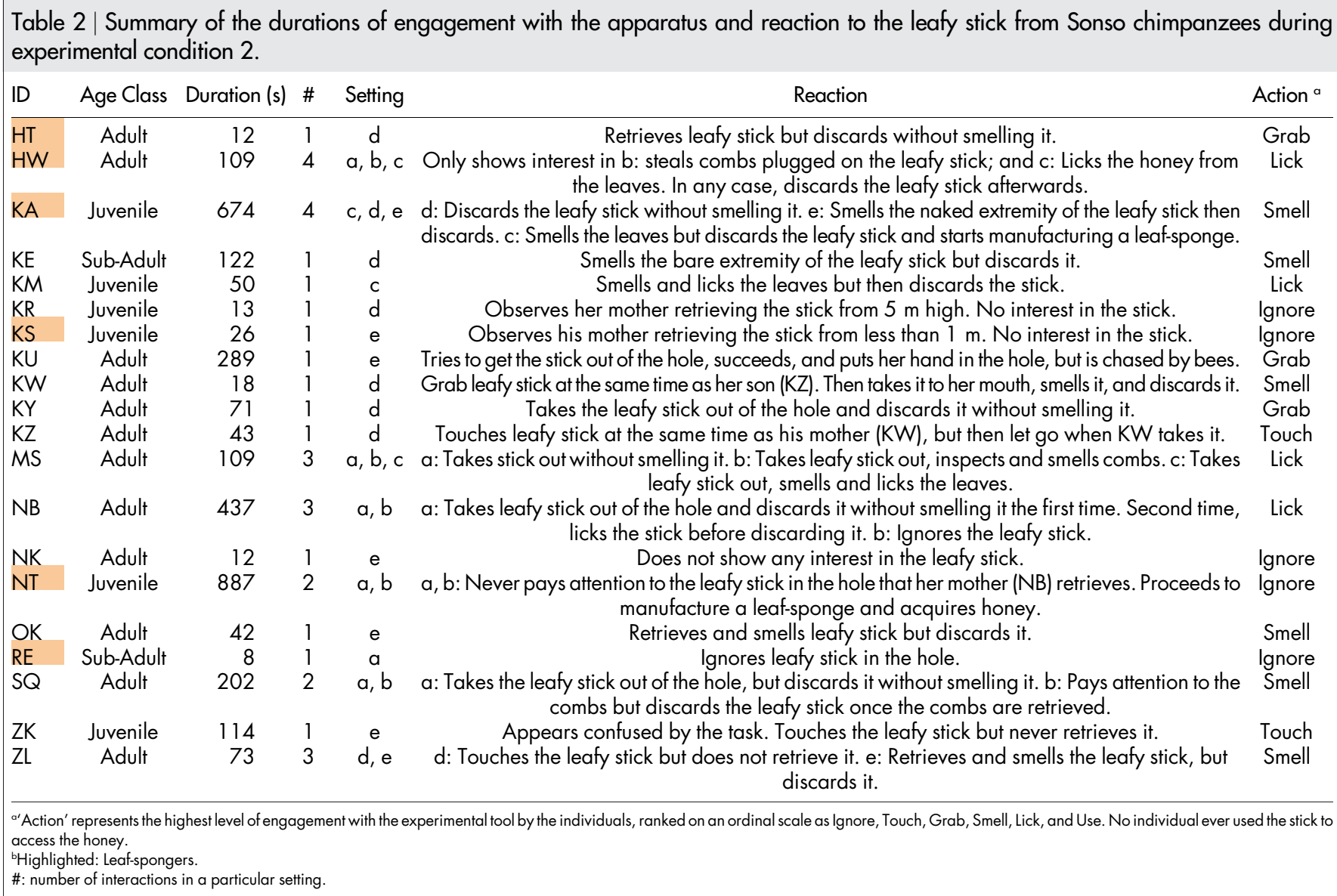


the three possible functions of the 'leafy stick' (sponging, brushing, dipping) and thus remained unsuccessful in their attempts to obtain honey. None of the leaf-spongers took advantage of the tool's two alternative functions.

\section{Discussion}

Our results are relevant to the study of chimpanzee cognition and culture and the links between the two phenomena. Experiment 1 shows that chimpanzees of two different communities with different (presumed) cultural backgrounds ${ }^{1}$ react differently to the affordances of an experimentally provided multi-functional tool. The stick-using Kanyawara chimpanzees found the stick part of the leafy stick most salient and useful to extract honey, while the non stickusing Sonso chimpanzees found the leafy part of the exact same tool most salient. Different communities of wild chimpanzees can thus react in radically different ways to the same features of their environment. This suggests that they may perceive and interpret their environment in different ways, notably in relation to their cultural knowledge. Similarly to our previous study ${ }^{20}$, the segregation of the behaviours chosen by the different communities was complete with no overlap. Chimpanzees at Kanyawara found the stick part of the tool the most salient, using it as a dipping device but ignoring the potential brushing and sponging possibilities. This was further illustrated by the behaviour of three individuals who removed and discarded all leaves from the leafy stick before inserting it into the honey. In contrast, the three chimpanzees at Sonso who engaged with the leafy stick focused on its leafy part, which was used to produce a leaf sponge to extract honey. In contrast, the wooden part of the tool was discarded. Out of the three, only one had already used a leaf-sponge in the baseline condition. The two new leaf-spongers had previously engaged with the apparatus (2009 experiment, obligatory condition) but had not produced any tool before. Although in one case, it may be due to the limited exposure to the apparatus the juvenile (KA) had during the baseline condition, it is less clear in the case of JT. A greater motivation to access the honey during this new session may explain why she manufactured a tool this time.

Generally, there was a trend for Kanyawara chimpanzees to engage longer with the apparatus than Sonso chimpanzees. However, we do not think that this is due to a difference in motivation to obtain honey, because successful tool-users at Sonso spent as much time engaging with the apparatus as successful tool-users at Kanyawara (table 1). Thus, the overall lower engagement time at Sonso is probably due to the numerous individuals who were unable to transfer their general leaf-sponging abilities to the problem at hand, which led them to abandon the apparatus more quickly than others. At Kanyawara, most individuals came up with a stick solution and success rates were thus much higher. In conclusion, wild chimpanzees appear to assess the functional properties of tools in community-specific ways, suggesting that cultural knowledge may impact on the way they comprehend their environment.

In experiment 2, we focused on the Sonso chimpanzees and exposed them to the technique used by the Kanyawara chimpanzees. This community was interesting for two reasons. Firstly, only seven of 29 individuals who engaged with the apparatus in this and the 2009 experiment came up with a tool solution, suggesting that transferring their general leaf-sponging skills to this problem was not an easy or automatic process. The same pattern was seen in experiment 1 where only two additional individuals used leaf-sponges to access the honey. Secondly, although 20 individuals showed much interest in the apparatus and engaged to different degrees with the leafy stick, none discovered the dipping or brushing functions of the tool. Therefore, these results do not support the idea that chimpanzees can learn tool use easily through affordance learning ${ }^{8,11}$, even if the functional properties are made highly conspicuous.

Our results suggest that previous experience may mediate what types of tools wild chimpanzees use in novel situations and perhaps more information, notably in terms of socially mediated input, may be needed before a novel tool behaviour can be learned. These findings are in line with research in captivity, which has shown that apes prefer to rely on previously acquired foraging techniques, with no evidence for modifications to improve efficiency, a phenomenon named 'conservatism ${ }^{23-24}$. However, observations in the wild suggest that chimpanzees can also adapt and modify an existing foraging technique, ant-fishing in trees, to some degree ${ }^{25}$. Why did the Sonso chimpanzees not discover the dipping behaviour? A first hypothesis is that they simply needed more exposure to the problem. In experiment 2 , most individuals only received 1 or 2 exposures, but never more than 4 , suggesting that more exposure may be necessary. This hypothesis is based on the assumption that the behaviour has already been present in the community, but that individuals need extended exposure to activate it. However, the point of our experiment was to address whether novel behaviour appears directly in response to favourable affordances, as proposed by Tennie et al. ${ }^{8}$. In this case, limited exposure is the natural condition because it is very unlikely that chimpanzees will encounter sticks protruding from beehives, a key condition for affordance learning. Our results, thus, do not support Tennie et al.'s ${ }^{8}$ model of how chimpanzees acquire tool use in the wild.

As outlined earlier, our data do not suggest that the Sonso chimpanzees' failure to acquire adequate tool use was due to a lack of motivation. Various additional observations also argue against a motivational account. Some individuals retrieved the stick and even licked the honey from it (NB), but they subsequently tried to access the honey with their hands without success. Another possible explanation for the lack of success is based on the notion of a sensitive phase. Perhaps the tested individuals did not develop tool use because they were simply too old ${ }^{26}$. This is not so likely because our sample of tested individuals ranged from young juveniles (e.g. NT) to old adults (e.g. NB), thus covering all ages. Finally, it is possible that several of the previously mentioned factors (sensitive phase, affordance learning, social settings) need to be present in combination. For example, it may be necessary for infant chimpanzees to encounter sticks left in cavities by their mothers and they may need to observe other group members performing actions associated with stick use in order to understand that sticks can be used as tools. There is evidence that more complicated tool use, such as termite fishing or nut cracking, are partly acquired by younger individuals when observing mothers and experienced individuals ${ }^{17,27-29}$.

Until the necessary research is done to elucidate the learning mechanisms of chimpanzee tool use in the wild we interpret our results in terms of a 'cultural bias', which constrains how chimpanzees of different communities perceive and evaluate their environment. Individuals acquire tool use in a socially-structured environment but individual trial-and-error learning most likely plays a key role ${ }^{17,30}$, which is consistent with the idea of hybrid learning ${ }^{18}$. In some ways, it does not matter what sorts of social learning processes have been at work to build the community-specific habits that subjects rely on to solve the task $\mathrm{k}^{31}$. Although habits can be acquired individually ${ }^{32}$, in chimpanzees this takes place in a social framework, giving each community its unique pattern and fulfilling therefore one of the main requirements of current definitions of animal culture ${ }^{33}$. The Sonso individuals simply did not see the affordances of the leafy stick as a dipping tool or a brush (and only a small number saw its functional properties as a sponge) probably because in this community tools are never used during food acquisition. Both Sonso and Kanyawara infants grow up in a world of leaves, twigs and branches, and both spend significant amounts of time playing with these objects, especially as infants (Gruber, personal observations ${ }^{34}$ ). Nevertheless, as adults, Kanyawara individuals reliably develop habitual stick use during food acquisition ${ }^{20}$ while Sonso individuals do not, suggesting that experiences during early ontogeny play a key role. Since sticks are not used in Sonso for food acquisition, grooming, 
resting or travelling, the main diurnal activities of wild chimpanzees ${ }^{35}$, nor in sexual interactions, they may generally become considered as irrelevant objects, a phenomenon that can have powerful inhibitory effects on learning (cf the 'learned irrelevance' effect ${ }^{36}$ ).

The apparent cultural bias observed in these chimpanzees shows some interesting parallels to what has been termed 'cultural override' in humans ${ }^{37}$. In spatial cognition experiments, Haun et al. showed that children learn to adopt their own culture's coordinate system, although they initially all use the same spatial coordinate system. Similarly, some parts of the environment appear to become irrelevant to chimpanzees during development, possibly because their mothers and other experienced individuals do not show any interest in them. Future research needs to address how exposure to other individuals' actions during infancy may determine which aspects they will perceive as salient in adulthood. In sum, our results suggest that there is an important cognitive dimension to observed behavioural variants in wild chimpanzees ${ }^{26}$ and that adopting a more cognitive approach to the study of culture in chimpanzees may bring new insights in understanding similarities with and differences from human cultures.

\section{Methods}

Subjects and Study Sites. The Sonso community $\left(01^{\circ} 43^{\prime} \mathrm{N}, 31^{\circ} 32^{\prime} \mathrm{E}\right)$ has been studied in the Budongo Forest since 1990 and has been fully habituated to human observers since 1994. At the time of the study, the community consisted of 69 individuals. The Kanyawara community $\left(00^{\circ} 33^{\prime} \mathrm{N}, 30^{\circ} 21^{\prime} \mathrm{E}\right)$ has been continuously studied in Kibale National Park since 1987 and has been fully habituated since 1994. At the time of the study, the community consisted of 46 individuals. The distance between the two sites is about $180 \mathrm{~km}$. Both Kanyawara and Sonso chimpanzees practice leaf-sponging on a customary basis, i.e. most members of the community show the behaviour ${ }^{1-2}$. Kanyawara chimpanzees can also be observed extracting fluids with sticks ('fluiddip') on a habitual basis, i.e. the behaviour has been observed several times in several individuals. Fluid-dip has allegedly been absent in Sonso over 20 years of continuous observation $^{22}$.

Set-up. Natural honey was acquired from local bee keepers of the Masindi District, Uganda, whose bees of the genus Apis foraged freely in Budongo Forest. We manufactured portable logs from fallen trees in their respective natural habitats. All logs were standardised to a length of $50 \mathrm{~cm}$ and a width of $25 \mathrm{~cm}$. We then drilled a rectangular-shaped hole of 4 by $5 \mathrm{~cm}$ (width $\times$ length) $16 \mathrm{~cm}$ deep in the middle of each log, using a manual drill (equal to the 'obligatory' condition in our previous study $\left.^{20}\right)$. Logs were positioned in locations frequently visited by the chimpanzees, such as on a pathway or near a feeding tree. At Sonso, logs were placed under Ficus sur trees, which are frequently visited by this community ${ }^{22,38}$. In Kanyawara, the log was placed under a Ficus natalensis tree, which was fruiting at the time of the experiment. Twigs, climbers, and leaves were available as potential raw material for tools in large quantities at both sites. Every morning, honey was poured into the hole by the experimenter (TG) to $10 \mathrm{~cm}$ below the surface. Additionally, honeycombs were used to cover the hole and protect it from insect invasion and to provide a conspicuous visual cue to the chimpanzees. A motion-sensitive video camera (PixController DVREye) was positioned to survey the hole and an immediate area of about $20 \mathrm{~m}^{2}$. All experiments were set up in the absence of chimpanzees. In Budongo, data were collected between February 20 and March 25, 2009 and September 14, 2009 and July 17, 2010. In Kibale, data were collected between April 2 and 22, 2009 and August 20 and 24, 2010.

Experimental conditions. Baseline condition (Sonso only). Chimpanzees were allowed to engage freely with the apparatus, with no tool present. In our previous study $^{20}$, Kanyawara chimpanzees had spent more time engaging with a similar apparatus, and they produced a stick tool after an average engagement time of $20 \mathrm{~s}$ in the 'obligatory' condition, i.e. when access to honey was only possible with the use of a tool ( $\mathrm{N}=11$ individuals). Some individuals tested at Sonso did not engage with the apparatus for $20 \mathrm{~s}$, and this opened the possibility that they simply did not have enough time to develop tool use. We addressed this by giving the Sonso individuals more time with the apparatus in the obligatory condition so that they matched the Kanyawara individuals in terms of mean engagement time. Because the main task in this experiment was identical to the obligatory condition in the previous 2009 experiment ${ }^{20}$, we established the baseline condition by combining the total engagement time for each individual. In both experiments, honey was enclosed in a small cavity that required inserting a tool to access it, although the dimensions of the substrate were very different (2009 study: large naturally fallen tree trunk; this study: $25 \mathrm{~cm} \times 50 \mathrm{~cm}$ portable $\log$ ). In the baseline condition we compared all individuals that had a combined engagement time with the apparatus of $>20 \mathrm{~s}$ when no tool was provided (table 1).

Experiment 1: Provisioning of the leafy stick. Chimpanzees at both sites were allowed to engage with the apparatus and a multi-functional tool, the 'leafy stick', which was positioned halfway between the hole and either edge of the log, randomly across trials. The tool was a $40 \mathrm{~cm}$ branch of Alstonia sp., a common shrub in Budongo and Kibale forests, stripped of all leaves on the lower $20 \mathrm{~cm}$ of its length. This way the leafy stick could be used either as a dipping device (by inserting the bare end), as a brush (by inserting the leafy end) or as a leaf-sponge (by removing and chewing its leaves and inserting the resulting sponge, figure 1). Skilled tool-users managed to access the honey in less than 10 minutes, by inserting the leafless end of the tool ('stick') or by removing the leaves and chewing them into a wadge ('sponge'). The two techniques appeared similar in efficiency although we could not test this statistically because the honey provided was rarely consumed entirely in Sonso as leaf-spongers were often displaced by more dominant individuals. However, a comparison of the average duration spent by the most keen tool-users at Sonso (NT, sponge) and Kanyawara QT, stick) during experiment 1 was $8.02 \mathrm{~min}(\mathrm{n}=2)$ and $8.21 \mathrm{~min}(\mathrm{n}=1)$.

With this experiment, our goal was to study if chimpanzees of different communities would find a particular part of the tool salient to use for the task, i.e. whether there were community-specific preferences, in line with the idea of a cultural bias in comprehending the environment. In contrast, if chimpanzees' selection of behaviour was only driven by environmental affordances, we would expect the chimpanzees to select the same behaviour to solve the task, independently of their community of origin.

Experiment 2: Highlighting the dipping function of the leafy stick (Sonso only). In the second experiment, we only tested the Sonso individuals because most of them ignored the tool provided in experiment 1 and remained unsuccessful in getting honey. The leafy stick was directly inserted into the hole before the chimpanzees arrival. This type of presentation was designed to make the alternative features of the tool particularly obvious because, when removing the tool from the hole, the honey would drip from the stick-part. For individuals who had previously responded with leaf-sponging, i.e. experienced tool-users, we were interested in whether they would consider these alternative functions of the tool, and favour any of them over of their existing leaf-sponging technique that they had transferred to this problem. For all other individuals, i.e. those who had never been observed manufacturing sponges or using other tools to extract resources, we were interested in whether this manipulation would make them learn stick use.

We tested five different settings to draw the attention of individuals to the dipping function of the tool: (a): stick part in cavity in contact with honey (dipping); (b): stick part in cavity in contact with honey, perforating through a comb (dipping); (c): leafy part in cavity in contact with honey (brushing); (d) stick part in cavity in contract with honey, but smaller $3 \times 3 \mathrm{~cm}$ hole; (e): stick part in cavity in contact with honey, with stick features highlighted by an adult baboon who engaged with the log before chimpanzees and chewed on the stick. We considered the possible health implications of this condition and concluded that it was sufficiently natural given that baboons and chimpanzees often forage in the same trees and thus, are subject to each other's pathogens on a frequent basis (see appendix 1). Each of these different settings thus highlighted the dipping function of the leafy stick in different ways.

The main goal of experiment 2 was to test whether the limited exposure to one of these settings could drive chimpanzees to develop stick use individually. It is not likely that Sonso chimpanzees ever encounter sticks left in natural bee-hives (because there are no other tool-using species in the forest that exploit honey). The experiment thus tested if affordance learning (and as such 'ecological' affordance), without any social influence, could trigger on its own the development of a certain cultural behaviour ('fluip-dip') in a naive chimpanzee community.

Ideally we would have tested all individuals in all settings. However, in field situations such experimental rigour is impossible to strive for, because experimenters have no control over individuals' travel decisions. As part of our commitment to keep the experimental manipulations inconspicuous, we never exposed a community for more than one week to a certain setting. We chose not conduct the complementary experiment at Kanyawara, i.e. providing a leaf-sponge next or in the hole, for health reasons. Leaf-sponges are produced by folding and chewing a bunch of leaves in the mouth, a manipulation that would have increased the risks of human pathogen transmissions.

Relevance of the comparative analysis and statistics. For the baseline condition, we compared the results of these experiments with results obtained in our previous study $^{20}$. We used a $20 \mathrm{~s}$ criterion in the baseline condition as described above. This was done to control for overall subject participation and exposure to this task and as a measure of motivation. We compared the total duration of engagement with the hole in both communities, especially before a tool was manufactured. In experiment 1 and 2 , however, given that chimpanzees in the two communities differed in their choices of behaviour towards the leafy stick in community-specific ways, we favoured a behavioural comparison and did not use any temporal criterion. All statistical tests were calculated with PASW Statistics v. 18.0.

1. Whiten, A. et al. Cultures in chimpanzees. Nature 399, 682-685 (1999).

2. Whiten, A. et al. Charting cultural variation in chimpanzees. Behaviour 138, 1481-1516 (2001).

3. Whiten, A. \& van Schaik, C. P. The evolution of animal 'cultures' and social intelligence. Philosophical Transactions of the Royal Society B: Biological Sciences 362 (2007).

4. McGrew, W. C. in The question of animal culture (eds K.N. Laland \& B.G. Galef) 41-69 (Harvard University Press, 2009). 
5. Boesch, C. Is culture a golden barrier between human and chimpanzee? Evolutionary Anthropology 12, 82-91 (2003).

6. Galef, B. G. in The question of animal culture (eds K.N. Laland \& B.G. Galef) 222 246 (Harvard University Press, 2009).

7. Tomasello, M. in The question of animal culture (eds K.N. Laland \& B.G. Galef) 198-221 (Harvard University Press, 2009).

8. Tennie, C., Call, J. \& Tomasello, M. Ratcheting up the ratchet: on the evolution of cumulative culture. Phil. Trans. R. Soc. B 364, 2045-2415 (2009).

9. Call, J. \& Tennie, C. Aninmal culture: Chimpanzee table manners? Current Biology 19, R981-R983 (2009).

10. Tomasello, M. in "Language" and intelligence in monkeys and apes: Comparative developmental perspectives (eds S. Parker \& K. Gibson) 274-311 (Cambridge University Press, 1990)

11. Tomasello, M. in Social learning in animals: The roots of culture (eds B. Galef \& C. Heyes) 319-346 (Academic Press, 1996)

12. Tomasello, M., Davis-Dasilva, M., Camak, L. \& Bard, K. Observational learning of tool-use by young chimpanzees. Human Evolution 2, 175-183 (1987).

13. Hopper, L. M., Lambeth, S. P., Schapiro, S. J. \& Whiten, A. Observational learning in chimpanzees and children studied through 'ghost' conditions. Proceedings of the Royal Society B: Biological Sciences 275, 835-840 (2008).

14. Tennie, C., Call, J. \& Tomasello, M. Evidence for emulation in chimpanzees in social settings using the floating peanut task. Plos ONE 5, e10544 (2010).

15. Hopper, L. M. et al. Experimental studies of traditions and underlying transmission processes in chimpanzees. Animal Behaviour 73, 1021-1032 (2007).

16. Laland, K. N. \& Galef, B. G. (eds) The question of animal culture (Harvard University Press, 2009).

17. Matsuzawa, T. et al. in Primate origin of human behavior and cognition (ed T. Matsuzawa) 557-574 (Springer, 2001).

18. Sterelny, K. The evolution and evolvability of culture. Mind \& Language 21, 137165 (2006)

19. Byrne, R. W. Commentary on Boesch, C. and Tomasello, M. Chimpanzee and human culture. Current Anthropology 39, 604-605 (1998).

20. Gruber, T., Muller, M. N., Strimling, P., Wrangham, R. W. \& Zuberbühler, K. Wild chimpanzees rely on cultural knowledge to solve an experimental honey acquisition task. Current Biology 19, 1806-1810 (2009).

21. Gruber, T. et al. The influence of ecology on chimpanzee cultural behaviour: A case study of 5 Ugandan chimpanzee communities. (submitted).

22. Reynolds, V. The chimpanzees of the Budongo forest: Ecology, behaviour and conservation. (Oxford University Press, 2005).

23. Marshall-Pescini, S. \& Whiten, A. Chimpanzees (Pan troglodytes) and the question of cumulative culture: An experimental approach. Animal Cognition 11, 449-456 (2008).

24. Hrubesch, C., Preuschoft, S. \& van Schaik, C. P. Skill mastery inhibits adoption of observed alternative solutions among chimpanzees (Pan troglodytes). Animal Cognition 12, 209-216 (2009).

25. Yamamoto, S., Yamakoshi, G., Humle, T. \& Matsuzawa, T. Invention and modification of a new tool use behavior: Ant-fishing in trees by a wild chimpanzee (Pan troglodytes verus) at Bossou, Guinea. American Journal of Primatology 70, 699-702 (2008).

26. Matsuzawa, T., Tomonaga, M. \& Tanaka, M. (eds) Cognitive development in chimpanzees (2006).

27. Lonsdorf, E. V., Everly, L. E. \& Pusey, A. E. Sex differences in learning in chimpanzees. Nature 428, 715-716 (2004).

28. Lonsdorf, E. V. Sex differences in the development of termite-fishing skills in the wild chimpanzees, Pan troglodytes schweinfurthii, of Gombe National Park, Tanzania. Animal Behaviour 70, 673-683 (2005).
29. Biro, D. et al. Cultural innovation and transmission of tool use in wild chimpanzees: Evidence from field experiments. Animal Cognition 6, 213-223. (2003)

30. Fragaszy, D. M. \& Visalberghi, E. Socially biased learning in monkeys. Learning \& Behavior 32, 24-35 (2004).

31. de Waal, F. B. M. \& Bonnie, K. E. in The question of animal culture (eds K.N. Laland \& B.G. Galef) 19-39 (Harvard University Press, 2009).

32. Pesendorfer, M. B. et al. The maintenance of traditions in marmosets: individual habit, not social conformity? A field experiment. Plos ONE 4, e4472 (2009).

33. Quiatt, D. \& Reynolds, V. Primate behaviour: Information, social knowledge, and the evolution of culture. (Cambridge University Press, 1993).

34. Kahlenberg, S. M. \& Wrangham, R. W. Sex differences in chimpanzees' use of sticks as play objects resemble those of children. Current Biology 20, R1067R1068 (2010).

35. Goodall, J. The chimpanzees of Gombe: Patterns of behavior. (Harvard University Press, 1986).

36. Mackintosh, N. J. The psychology of animal learning. (Academic Press, 1974).

37. Haun, D. B. M., Rapold, C. J., Call, J., Janzen, G. \& Levinson, S. C. Cognitive cladistics and cultural override in Hominid spatial cognition. Proceedings of the National Academy of Sciences of the United States of America 103, 17568-17573 (2006).

38. Newton-Fisher, N. E. The diet of chimpanzees in the Budongo Forest Reserve, Uganda. African Journal of Ecology 37, 344-354 (1999).

\section{Acknowledgements}

Permission to conduct research was given by Uganda Wildlife Authority (UWA), Ugandan National Council for Science and Technology (UNCST) and National Forestry Authority (NFA). Ethical approval was given by the School of Psychology, University of St Andrews. We are grateful to the Royal Zoological Society of Scotland for providing core funding for the Budongo Conservation Field Station. Research at Kibale was supported by a grant from the U.S. National Science Foundation (award 0416125). We are especially grateful to Stephen Amati, James Kyomuhendo, and Emily Otali for help with fieldwork. The study was supported by the Leverhulme Trust (UK). We commemorate the Congolese workers in the oil palm plantation owned by Lord Leverhulme.

\section{Author Contributions}

T.G and K.Z: designed research; T.G performed research and analysed data; T.G and K.Z wrote the paper; M.N.M., R.W. and V.R. contributed technical expertise. All co-authors reviewed the paper.

\section{Additional information}

Supplementary information accompanies this paper at http://www.nature.com/ scientificreports

Competing financial interests The authors declare no competing financial interests.

License: This work is licensed under a Creative Commons

Attribution-NonCommercial-NoDerivative Works 3.0 Unported License. To view a copy of this license, visit http://creativecommons.org/licenses/by-nc-nd/3.0/

How to cite this article: Gruber, T., Muller, M.N., Reynolds, V., Wrangham, R. \& Zuberbühler, K. Community-specific evaluation of tool affordances in wild chimpanzees. Sci. Rep. 1, 128; DOI:10.1038/srep00128 (2011) 